\title{
Hyperekplexia: a surprise diagnosis
}

\author{
Özlem Yayıcı Köken ${ }^{1}$, Özge Toptaş Dedeoğlu², Ayşe Aksoy ${ }^{3}$, Deniz Yüksel ${ }^{4}$ \\ ${ }^{1}$ Ankara City Hospital, Children's Hospital, Department of Pediatric Neurology, Ankara, Turkey \\ ${ }^{2}$ Mardin State Hospital, Department of Pediatric Neurology, Mardin, Turkey \\ ${ }^{3}$ Samsun Ondokuz Mayıs University, Department of Pediatric Neurology, Samsun, Turkey \\ ${ }^{4}$ University of Health Sciences, Dr. Sami Ulus Maternity and Children's Health and Diseases Training and Research Hospital, \\ Department of Pediatric Neurology, Ankara, Turkey
}

Key words: hyperekplexia, SIDS, startle disease

(Neurol Neurochir Pol 2020; 54 (5): 473-474)

\section{To the Editors:}

Hyperekplexia, which is also known as startle disease, is a very rare paroxysmal neurogenetic disorder. It is also one cause of Sudden Infant Death Syndrome (SIDS). Hypertonicity and exaggerated startle reflex caused by visual, auditory or tactile stimuli in the newborn are the key clinical findings of hyperekplexia [1]. Molecular genetic studies have revealed that mutations in the genes coding postsynaptic proteins and the glycine receptors which control postsynaptic inhibition in the spinal cord and other regions of the central nervous system are responsible for this disease $[2,3]$. The disease has been primarily and most frequently associated with a mutation in the gene that codes the alpha-1 subunit of the glycine receptor which is located on 5q33.1. It has been associated with heterozygous, homozygous or compound heterozygous mutations in the GLRA1 gene which can exhibit autosomal dominant or recessive traits, and has been subgrouped as hyperekplexia- 1 in the literature [4].

We here report the case of a two-month-old girl with a prediagnosis of hyperekplexia due to clinical and electrophysiological features, who was subsequently diagnosed with hyperekplexia as a result of target gene analysis which revealed a deletion in the GLRA1 gene. The patient, who had been on antiepileptic medicine since she was two weeks old, was admitted due to startling and jerking caused by unexpected sounds and touching which occurred both when awake and when asleep, and were first seen in the first postnatal week. She was born via spontaneous vaginal delivery at the end of a non-problematic pregnancy to parents who were third degree relatives. Family history was negative for hyperekplexia and SIDS. She had a high frequency, low amplitude tremor accompanied by tonic contractions of the upper and lower extremities which was first noticed when she was one week old, and was also observed during neurological examination. Her parents stated that these tremors happened 20-25 times a day following auditory and tactile stimuli, and were often accompanied by perioral cyanosis.

She had been seen by a paediatric neurologist and was first given phenobarbital (6 $\mathrm{mg} / \mathrm{kg} /$ day divided into two doses) but levetiracetam $(30 \mathrm{mg} / \mathrm{kg} /$ day divided into two doses) was added when she did not respond to that treatment. Video electroencephalography (V-EEG) revealed a specific recording simultaneous to tonic contractions throughout the body which were caused by a tactile stimulus.

The recording had rhythmic, monomorphic spikes in the beta frequency which abruptly initiated from the posterior parts of bilateral hemispheres and were constant and did not change or evolve. These waves were abruptly terminated simultaneous to the termination of tonic seizures and showed generalised background slowing, which lasted for 2-3 seconds in the EEG (Fig. 1). Cranial magnetic resonance imaging (MRI) and metabolic panel (liver and kidney function tests, tandem mass, organic acids, pyruvate and lactate) were within normal limits.

Therefore the patient aged two months was diagnosed with hyperekplexia based on the occurrence of all episodes with stimulus and video electroencephalographic features. All antiepileptic treatment was stopped, and clonazepam treatment was initiated. Clonazepam was given in three divided 


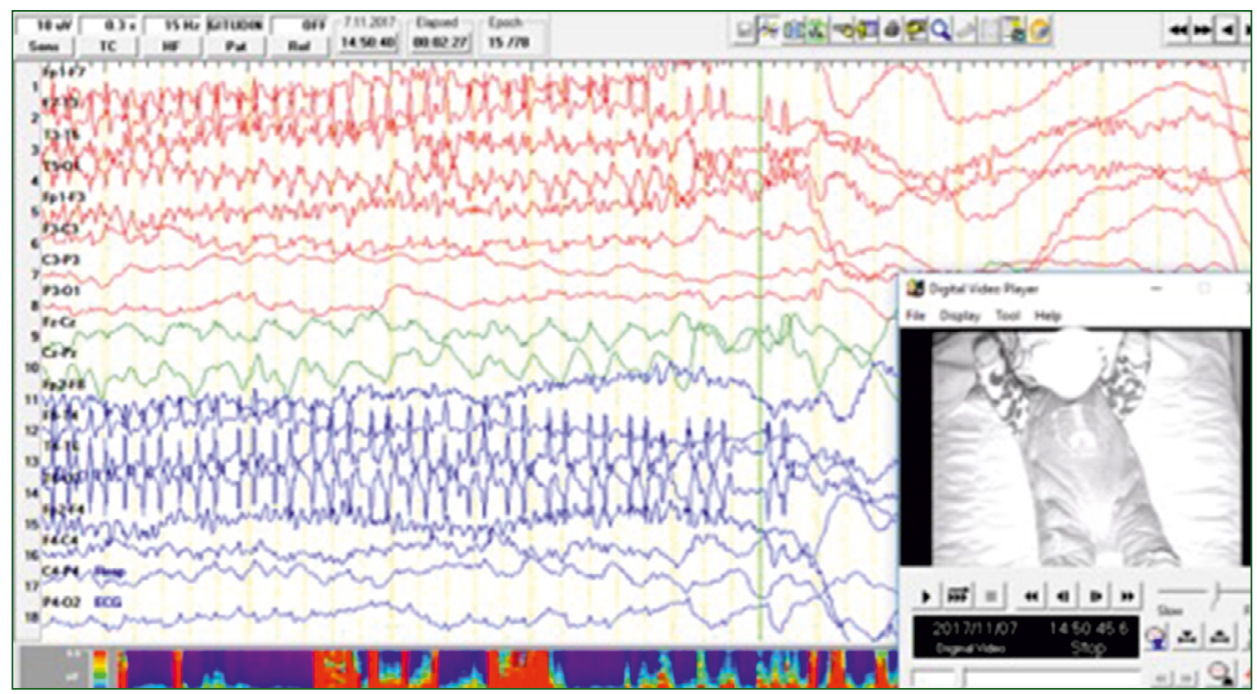

Figure 1. EEG findings of the patient during tonic contractions

doses starting with $0.05 \mathrm{mg} / \mathrm{kg} /$ day and increased to the target dose of $0.15 \mathrm{mg} / \mathrm{kg} /$ day. A dramatic response to clonazepam treatment was observed.

A genetic study targeted at the GLRA1 gene for the prediagnosis of hyperekplexia was carried out, and a homozygous deletion between exons 1-7 was found. The GLRA-1 gene has nine exons, and the homozygous deletion in our patient was consistent with a complete loss of gene function. The diagnosis was confirmed by a molecular genetic study which was performed in the third month of life, and the family was offered genetic counselling regarding future pregnancies.

In the last follow up, which was performed when the patient was under clonazepam treatment and was six months old, the tonic spasms had completely disappeared although minor jerks following tactile stimuli had not. There were no side effects of the treatment and the patient's development was normal, despite the cases reported in the literature with delayed motor development [5]. Also, there is no history of apnoea attacks.

Hyperekplexia is a rare non-epileptic phenomenon which clinicians often mistake for epilepsy in their daily practice. Hyperekplexia is known to be caused by a variety of genes encoding both pre- and postsynaptic proteins. The symptoms displayed, as well as the forms of heritance, vary according to which gene is affected.

In terms of the type of mutation in the GLRA1 gene, it should also be noted that deletion is extremely rare in the reported cases. It is very important to recognise the specific clinical features, to evaluate electroencephalographic patterns in conjunction with clinical findings, and to offer counselling to the families about responses to therapy and the genetic traits of this disease. As a result, hyperekplexia is a neurogenetic disorder which must be borne in mind by general practitioners, paediatricians, and paediatric neurologists.

\section{References}

1. Suhren 0, Bruyn GW, Tuynman JA. Hyperexplexia. Journal of the Neurological Sciences. 1966; 3(6): 577-605, doi: 10.1016/0022510x(66)90047-5.

2. Davies JS, Chung SK, Thomas RH, et al. The glycinergic system in human startle disease: a genetic screening approach. Front Mol Neurosci. 2010; 3: 8, doi: 10.3389/fnmol.2010.00008, indexed in Pubmed: 20407582.

3. Harvey RJ, Topf M, Harvey K, et al. The genetics of hyperekplexia: more than startle! Trends Genet. 2008; 24(9): 439-447, doi: 10.1016/j. tig.2008.06.005, indexed in Pubmed: 18707791.

4. Shiang R, Ryan SG, Zhu YZ, et al. Mutations in the alpha 1 subunit of the inhibitory glycine receptor cause the dominant neurologic disorder, hyperekplexia. Nat Genet. 1993; 5(4): 351-358, doi: 10.1038/ ng1293-351, indexed in Pubmed: 8298642.

5. Thomas RH, Chung SK, Wood SE, et al. Genotype-phenotype correlations in hyperekplexia: apnoeas, learning difficulties and speech delay. Brain. 2013; 136(Pt 10): 3085-3095, doi: 10.1093/brain/awt207, indexed in Pubmed: 24030948. 